Bull. Austral. Math. Soc.

$46 \mathrm{C} 05,26 \mathrm{D} 15$

VoL. $72(2005)$ [337-347]

\title{
BOUNDS FOR THE DISTANCE TO FINITE-DIMENSIONAL SUBSPACES
}

\section{S.S. DRAGOMIR}

\begin{abstract}
We establish upper bounds for the distance to finite-dimensional subspaces in inner product spaces and improve some generalisations of Bessel's inequality obtained by Boas, Bellman and Bombieri. Refinements of the Hadamard inequality for Gram determinants are also given.
\end{abstract}

\section{INTRODUCTION}

Let $(H ;\langle\cdot, \cdot\rangle)$ be an inner product space over the real or complex number field $\mathbb{K},\left\{y_{1}, \ldots, y_{n}\right\}$ a subset of $H$ and $G\left(y_{1}, \ldots, y_{n}\right)$ the gram matrix of $\left\{y_{1}, \ldots, y_{n}\right\}$ where $(i, j)$-entry is $\left\langle y_{i}, y_{j}\right\rangle$. The determinant of $G\left(y_{1}, \ldots, y_{n}\right)$ is called the Gram determinant of $\left\{y_{1}, \ldots, y_{n}\right\}$ and is denoted by $\Gamma\left(y_{1}, \ldots, y_{n}\right)$. Thus,

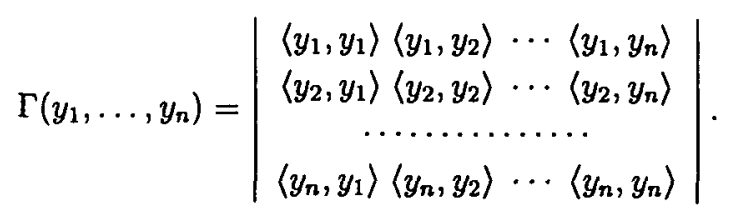

Following [4, p. 129-133], we state here some general results for the Gram determinant that will be used in the sequel.

(1) Let $\left\{x_{1}, \ldots, x_{n}\right\} \subset H$. Then $\Gamma\left(x_{1}, \ldots, x_{n}\right) \neq 0$ if and only if $\left\{x_{1}, \ldots, x_{n}\right\}$ is linearly independent;

(2) Let $M=\operatorname{span}\left\{x_{1}, \ldots, x_{n}\right\}$ be $n$-dimensional in $H$, that is, $\left\{x_{1}, \ldots, x_{n}\right\}$ is linearly independent. Then for each $x \in H$, the distance $d(x, M)$ from $x$ to the linear subspace $H$ has the representations

$$
d^{2}(x, M)=\frac{\Gamma\left(x_{1}, \ldots, x_{n}, x\right)}{\Gamma\left(x_{1}, \ldots, x_{n}\right)}
$$

and

$$
d^{2}(x, M)=\|x\|^{2}-\beta^{T} G^{-1} \beta
$$

Received 17th January, 2005

Copyright Clearance Centre, Inc. Serial-fee code: 0004-9727/05 \$A2.00+0.00. 
where $G=G\left(x_{1}, \ldots, x_{n}\right), G^{-1}$ is the inverse matrix of $G$ and

$$
\beta^{T}=\left(\left\langle x, x_{1}\right\rangle,\left\langle x, x_{2}\right\rangle, \ldots,\left\langle x, x_{n}\right\rangle\right),
$$

denotes the transpose of the column vector $\beta$.

Moreover, one has the simpler representation

$$
d^{2}(x, M)= \begin{cases}\|x\|^{2}-\frac{\left(\sum_{i=1}^{n}\left|\left\langle x, x_{i}\right\rangle\right|^{2}\right)^{2}}{\left\|\sum_{i=1}^{n}\left\langle x, x_{i}\right\rangle x_{i}\right\|^{2}} & \text { if } x \notin M^{\perp} \\ \|x\|^{2} & \text { if } x \in M^{\perp}\end{cases}
$$

where $M^{\perp}$ denotes the orthogonal complement of $M$.

(3) Let $\left\{x_{1}, \ldots, x_{n}\right\}$ be a set of nonzero vectors in $H$. Then

$$
0 \leqslant \Gamma\left(x_{1}, \ldots, x_{n}\right) \leqslant\left\|x_{1}\right\|^{2}\left\|x_{2}\right\|^{2} \cdots\left\|x_{n}\right\|^{2} .
$$

The equality holds on the left (respectively right) side of (1.4) if and only if $\left\{x_{1}, \ldots, x_{n}\right\}$ is linearly dependent (respectively orthogonal). The first inequality in (1.4) is known in the literature as Gram's inequality while the second one is known as Hadamard's inequality.

(4) If $\left\{x_{1}, \ldots, x_{n}\right\}$ is an orthonormal set in $H$, that is, $\left\langle x_{i}, x_{j}\right\rangle=\delta_{i j}, i, j$ $\in\{1, \ldots, n\}$, where $\delta_{i j}$ is Kronecker's delta, then

$$
d^{2}(x, M)=\|x\|^{2}-\sum_{i=1}^{n}\left|\left\langle x, x_{i}\right\rangle\right|^{2}
$$

- The following inequalities which involve Gram determinants may be stated as well [9, p. 597]:

$$
\begin{aligned}
\frac{\Gamma\left(x_{1}, \ldots, x_{n}\right)}{\Gamma\left(x_{1}, \ldots, x_{k}\right)} \leqslant & \frac{\Gamma\left(x_{2}, \ldots, x_{n}\right)}{\Gamma\left(x_{1}, \ldots, x_{k}\right)} \leqslant \cdots \leqslant \Gamma\left(x_{k+1}, \ldots, x_{n}\right) \\
& \Gamma\left(x_{1}, \ldots, x_{n}\right) \leqslant \Gamma\left(x_{1}, \ldots, x_{k}\right) \Gamma\left(x_{k+1}, \ldots, x_{n}\right)
\end{aligned}
$$

and

$$
\Gamma^{1 / 2}\left(x_{1}+y_{1}, x_{2}, \ldots, x_{n}\right) \leqslant \Gamma^{1 / 2}\left(x_{1}, x_{2}, \ldots, x_{n}\right)+\Gamma^{1 / 2}\left(y_{1}, x_{2}, \ldots, x_{n}\right) .
$$

The main aim of this paper is to point out some upper bounds for the distance $d(x, M)$ in terms of the linearly independent vectors $\left\{x_{1}, \ldots, x_{n}\right\}$ that $\operatorname{span} M$ and $x \notin M^{\perp}$, where $M^{\perp}$ is the orthogonal complement of $M$ in the inner product space $(H ;\langle\cdot, \cdot\rangle)$.

As a by-product of this endeavour, some refinements of the generalisations for Bessel's inequality due to several authors including: Boas, Bellman and Bombieri are obtained. Refinements for the well known Hadamard's inequality for Gram determinants are also derived. 


\section{UPPER BOUNDS FOR $d(x, M)$}

The following result may be stated.

THEOREM 1. Let $\left\{x_{1}, \ldots, x_{n}\right\}$ be a linearly independent system of vectors in $H$ and $M:=\operatorname{span}\left\{x_{1}, \ldots, x_{n}\right\}$. If $x \notin M^{\perp}$, then

$$
d^{2}(x, M)<\frac{\|x\|^{2} \sum_{i=1}^{n}\left\|x_{i}\right\|^{2}-\sum_{i=1}^{n}\left|\left\langle x, x_{i}\right\rangle\right|^{2}}{\sum_{i=1}^{n}\left\|x_{i}\right\|^{2}}
$$

or, equivalently,

$$
\Gamma\left(x_{1}, \ldots, x_{n}, x\right)<\frac{\|x\|^{2} \sum_{i=1}^{n}\left\|x_{i}\right\|^{2}-\sum_{i=1}^{n}\left|\left\langle x, x_{i}\right\rangle\right|^{2}}{\sum_{i=1}^{n}\left\|x_{i}\right\|^{2}} \cdot \Gamma\left(x_{1}, \ldots, x_{n}\right) .
$$

PROOF: If we use the Cauchy-Bunyakovsky-Schwarz type inequality

$$
\left\|\sum_{i=1}^{n} \alpha_{i} y_{i}\right\|^{2} \leqslant \sum_{i=1}^{n}\left|\alpha_{i}\right|^{2} \sum_{i=1}^{n}\left\|y_{i}\right\|^{2}
$$

that can be easily deduced from the obvious identity

$$
\sum_{i=1}^{n}\left|\alpha_{i}\right|^{2} \sum_{i=1}^{n}\left\|y_{i}\right\|^{2}-\left\|\sum_{i=1}^{n} \alpha_{i} y_{i}\right\|^{2}=\frac{1}{2} \sum_{i, j=1}^{n}\left\|\overline{\alpha_{i}} x_{j}-\overline{\alpha_{j}} x_{i}\right\|^{2},
$$

we can state that

$$
\left\|\sum_{i=1}^{n}\left\langle x, x_{i}\right\rangle x_{i}\right\|^{2} \leqslant \sum_{i=1}^{n}\left|\left\langle x, x_{i}\right\rangle\right|^{2} \sum_{i=1}^{n}\left\|x_{i}\right\|^{2} .
$$

Note that the equality case holds in (2.5) if and only if, by (2.4),

$$
\overline{\left\langle x, x_{i}\right\rangle} x_{j}=\overline{\left\langle x, x_{i}\right\rangle} x_{i}
$$

for each $i, j \in\{1, \ldots, n\}$.

Utilising the expression (1.3) of the distance $d(x, M)$, we have

$$
d^{2}(x, M)=\|x\|^{2}-\frac{\sum_{i=1}^{n}\left|\left\langle x, x_{i}\right\rangle\right|^{2} \sum_{i=1}^{n}\left\|x_{i}\right\|^{2}}{\left\|\sum_{i=1}^{n}\left\langle x, x_{i}\right\rangle x_{i}\right\|^{2}} \cdot \frac{\sum_{i=1}^{n}\left|\left\langle x, x_{i}\right\rangle\right|^{2}}{\sum_{i=1}^{n}\left\|x_{i}\right\|^{2}} .
$$

Since $\left\{x_{1}, \ldots, x_{n}\right\}$ are linearly independent, hence (2.6) cannot be achieved and then we have strict inequality in (2.5).

Finally, on using (2.5) and (2.7) we get the desired result (2.1).

REMARK 1. It is known that (see (1.4)) if not all $\left\{x_{1}, \ldots, x_{n}\right\}$ are orthogonal to each other, then the following result which is well known in the literature as Hadamard's inequality holds:

$$
\Gamma\left(x_{1}, \ldots, x_{n}\right)<\left\|x_{1}\right\|^{2}\left\|x_{2}\right\|^{2} \cdots\left\|x_{n}\right\|^{2} .
$$


Utilising the inequality (2.2), we may write successively:

$$
\begin{aligned}
\Gamma\left(x_{1}, x_{2}\right) \leqslant & \frac{\left\|x_{1}\right\|^{2}\left\|x_{2}\right\|^{2}-\left|\left\langle x_{2}, x_{1}\right\rangle\right|^{2}}{\left\|x_{1}\right\|^{2}}\left\|x_{1}\right\|^{2} \leqslant\left\|x_{1}\right\|^{2}\left\|x_{2}\right\|^{2}, \\
\Gamma\left(x_{1}, x_{2}, x_{3}\right)< & \frac{\left\|x_{3}\right\|^{2} \sum_{i=1}^{2}\left\|x_{i}\right\|^{2}-\sum_{i=1}^{2}\left|\left\langle x_{3}, x_{i}\right\rangle\right|^{2}}{\sum_{i=1}^{2}\left\|x_{i}\right\|^{2}} \Gamma\left(x_{1}, x_{2}\right) \\
\leqslant & \left\|x_{3}\right\|^{2} \Gamma\left(x_{1}, x_{2}\right), \\
& \ldots \ldots \ldots \ldots \ldots \ldots \ldots \ldots \ldots \ldots \ldots \ldots \ldots \ldots \ldots \ldots \ldots \\
\Gamma\left(x_{1}, \ldots, x_{n-1}, x_{n}\right)< & \frac{\left\|x_{n}\right\|^{2} \sum_{i=1}^{n-1}\left\|x_{i}\right\|^{2}-\sum_{i=1}^{n-1}\left|\left\langle x_{n}, x_{i}\right\rangle\right|^{2}}{\sum_{i=1}^{n-1}\left\|x_{i}\right\|^{2}} \Gamma\left(x_{1}, \ldots, x_{n-1}\right) \\
\leqslant & \left\|x_{n}\right\|^{2} \Gamma\left(x_{1}, \ldots, x_{n-1}\right) .
\end{aligned}
$$

Multiplying the above inequalities, we deduce

$$
\begin{aligned}
\Gamma\left(x_{1}, \ldots, x_{n-1}, x_{n}\right) & <\left\|x_{1}\right\|^{2} \prod_{k=2}^{n}\left(\left\|x_{k}\right\|^{2}-\frac{1}{\sum_{i=1}^{k-1}\left\|x_{i}\right\|^{2}} \sum_{i=1}^{k-1}\left|\left\langle x_{k}, x_{i}\right\rangle\right|^{2}\right) \\
& \leqslant \prod_{j=1}^{n}\left\|x_{j}\right\|^{2}
\end{aligned}
$$

valid for a system of $n \geqslant 2$ linearly independent vectors which are not orthogonal on each other.

In [7], the author has obtained the following inequality.

LEMMA 1. Let $z_{1}, \ldots, z_{n} \in H$ and $\alpha_{1}, \ldots, \alpha_{n} \in \mathbb{K}$. Then one has the inequalities:

$$
\begin{aligned}
&\left\|\sum_{i=1}^{n} \alpha_{i} z_{i}\right\|^{2} \leqslant\left\{\begin{array}{l}
\max _{1 \leqslant i \leqslant n}\left|\alpha_{i}\right|^{2} \sum_{i=1}^{n}\left\|z_{i}\right\|^{2} ; \\
\left(\sum_{i=1}^{n}\left|\alpha_{i}\right|^{2 \alpha}\right)^{1 / \alpha}\left(\sum_{i=1}^{n}\left\|z_{i}\right\|^{2 \beta}\right)^{1 / p} \\
\sum_{i=1}^{n}\left|\alpha_{i}\right|^{2} \max _{1 \leqslant i \leqslant n}\left\|z_{i}\right\|^{2} ; \\
+
\end{array}\right. \\
&\left\{\begin{array}{l}
{\left[\max _{1 \leqslant i \neq j \leqslant n}\left\{\left|\alpha_{i} \alpha_{j}\right|\right\} \sum_{1 \leqslant i \neq j \leqslant n}\left|\left\langle z_{i}, z_{j}\right\rangle\right| ;\right.} \\
\left.\left[\sum_{i=1}^{n}\left|\alpha_{i}\right|^{\gamma}\right)^{2}-\sum_{i=1}^{n}\left|\alpha_{i}\right|^{2 \gamma}\right]^{1 / \gamma}\left(\sum_{1 \leqslant i \neq j \leqslant n}\left|\left\langle z_{i}, z_{j}\right\rangle\right|^{\delta}\right)^{1 / \delta}
\end{array}\right. \\
& {\left[\left(\sum_{i=1}^{n}\left|\alpha_{i}\right|\right)^{2}-\sum_{i=1}^{n}\left|\alpha_{i}\right|^{2}\right]_{1 \leqslant i \neq j \leqslant n}\left|\left\langle z_{i}, z_{j}\right\rangle\right| ; }
\end{aligned}
$$

where any term in the first branch can be combined with each term from the second branch giving 9 possible combinations. 
Out of these, we select the following ones that are of relevance for further consideration:

$$
\begin{aligned}
\left\|\sum_{i=1}^{n} \alpha_{i} z_{i}\right\|^{2} & \leqslant \max _{1 \leqslant i \leqslant n}\left\|z_{i}\right\|^{2} \sum_{i=1}^{n}\left|\alpha_{i}\right|^{2}+\max _{1 \leqslant i<j \leqslant n}\left|\left\langle z_{i}, z_{j}\right\rangle\right|\left[\left(\sum_{i=1}^{n}\left|\alpha_{i}\right|\right)^{2}-\sum_{i=1}^{n}\left|\alpha_{i}\right|^{2}\right] \\
& \leqslant \sum_{i=1}^{n}\left|\alpha_{i}\right|^{2}\left(\max _{1 \leqslant i \leqslant n}\left\|z_{i}\right\|^{2}+(n-1) \max _{1 \leqslant i<j \leqslant n}\left|\left\langle z_{i}, z_{j}\right\rangle\right|\right)
\end{aligned}
$$

and

$$
\begin{aligned}
\left\|\sum_{i=1}^{n} \alpha_{i} z_{i}\right\|^{2} & \leqslant \max _{1 \leqslant i \leqslant n}\left\|z_{i}\right\|^{2} \sum_{i=1}^{n}\left|\alpha_{i}\right|^{2}+\left[\left(\sum_{i=1}^{n}\left|\alpha_{i}\right|^{2}\right)^{2}-\sum_{i=1}^{n}\left|\alpha_{i}\right|^{4}\right]^{1 / 2} \\
& \times\left(\sum_{1 \leqslant i \neq j \leqslant n}\left|\left\langle z_{i}, z_{j}\right\rangle\right|^{2}\right)^{1 / 2} \\
& \leqslant \sum_{i=1}^{n}\left|\alpha_{i}\right|^{2}\left[\max _{1 \leqslant i \leqslant n}\left\|z_{i}\right\|^{2}+\left(\sum_{1 \leqslant i \neq j \leqslant n}\left|\left\langle z_{i}, z_{j}\right\rangle\right|^{2}\right)^{1 / 2}\right] .
\end{aligned}
$$

Note that the last inequality in (2.11) follows by the fact that

$$
\left(\sum_{i=1}^{n}\left|\alpha_{i}\right|\right)^{2} \leqslant n \sum_{i=1}^{n}\left|\alpha_{i}\right|^{2}
$$

while the last inequality in (2.12) is obvious.

Utilising the above inequalities (2.11) and (2.12) which provide alternatives to the Cauchy-Bunyakovsky-Schwarz inequality (2.3), we can state the following results.

TheOREM 2. Let $\left\{x_{1}, \ldots, x_{n}\right\}, M$ and $x$ be as in Theorem 1. Then

$$
d^{2}(x, M) \leqslant \frac{\|x\|^{2}\left[\max _{1 \leqslant i \leqslant n}\left\|x_{i}\right\|^{2}+\left(\sum_{1 \leqslant i \neq j \leqslant n}\left|\left\langle x_{i}, x_{j}\right\rangle\right|^{2}\right)^{1 / 2}\right]-\sum_{i=1}^{n}\left|\left\langle x, x_{i}\right\rangle\right|^{2}}{\max _{1 \leqslant i \leqslant n}\left\|x_{i}\right\|^{2}+\left(\sum_{1 \leqslant i \neq j \leqslant n}\left|\left\langle x_{i}, x_{j}\right\rangle\right|^{2}\right)^{1 / 2}}
$$

or, equivalently,

$$
\begin{array}{r}
\Gamma\left(x_{1}, \ldots, x_{n}, x\right) \\
\leqslant \frac{\|x\|^{2}\left[\max _{1 \leqslant i \leqslant n}\left\|x_{i}\right\|^{2}+\left(\sum_{1 \leqslant i \neq j \leqslant n}\left|\left\langle x_{i}, x_{j}\right\rangle\right|^{2}\right)^{1 / 2}\right]-\sum_{i=1}^{n}\left|\left\langle x, x_{i}\right\rangle\right|^{2}}{\max _{1 \leqslant i \leqslant n}\left\|x_{i}\right\|^{2}+\left(\sum_{1 \leqslant i \neq j \leqslant n}\left|\left\langle x_{i}, x_{j}\right)\right|^{2}\right)^{1 / 2}} \\
\times \Gamma\left(x_{1}, \ldots, x_{n}\right) .
\end{array}
$$

Proof: Utilising the inequality (2.12) for $\alpha_{i}=\left\langle x, x_{i}\right\rangle$ and $z_{i}=x_{i}, i \in\{1, \ldots, n\}$, we can write:

$$
\left\|\sum_{i=1}^{n}\left\langle x, x_{i}\right\rangle x_{i}\right\|^{2} \leqslant \sum_{i=1}^{n}\left|\left\langle x, x_{i}\right\rangle\right|^{2}\left[\max _{1 \leqslant i \leqslant n}\left\|x_{i}\right\|^{2}+\left(\sum_{1 \leqslant i \neq j \leqslant n}\left|\left\langle x_{i}, x_{j}\right\rangle\right|^{2}\right)^{1 / 2}\right]
$$


for any $x \in H$.

Now, since, by the representation formula (1.3)

$$
d^{2}(x, M)=\|x\|^{2}-\frac{\sum_{i=1}^{n}\left|\left\langle x, x_{i}\right\rangle\right|^{2}}{\left\|\sum_{i=1}^{n}\left\langle x, x_{i}\right\rangle x_{i}\right\|^{2}} \cdot \sum_{i=1}^{n}\left|\left\langle x, x_{i}\right\rangle\right|^{2},
$$

for $x \notin M^{\perp}$, hence, by (2.15) and (2.16) we deduce the desired result (2.13).

REMARK 2. In 1941, Boas [2] and in 1944, Bellman [1], independent of each other, proved the following generalisation of Bessel's inequality:

$$
\sum_{i=1}^{n}\left|\left\langle y, y_{i}\right\rangle\right|^{2} \leqslant\|y\|^{2}\left[\max _{1 \leqslant i \leqslant n}\left\|y_{i}\right\|^{2}+\left(\sum_{1 \leqslant i \neq j \leqslant n}\left|\left\langle y_{i}, y_{j}\right\rangle\right|^{2}\right)^{1 / 2}\right],
$$

provided $y$ and $y_{i}(i \in\{1, \ldots, n\})$ are arbitrary vectors in the inner product space $(H ;\langle\cdot, \cdot\rangle)$. If $\left\{y_{i}\right\}_{i \in\{1, \ldots, n\}}$ are orthonormal, then $(2.17)$ reduces to Bessel's inequality.

In this respect, one may see (2.13) as a refinement of the Boas-Bellman result (2.17).

REMARK 3. On making use of a similar argument to that utilised in Remark 1, one can obtain the following refinement of the Hadamard inequality:

$$
\begin{aligned}
\Gamma\left(x_{1}, \ldots, x_{n}\right) & \leqslant\left\|x_{1}\right\|^{2} \prod_{k=2}^{n}\left(\left\|x_{k}\right\|^{2}-\frac{\sum_{i=1}^{k-1}\left|\left\langle x_{k}, x_{i}\right\rangle\right|^{2}}{\max _{1 \leqslant i \leqslant k-1}\left\|x_{i}\right\|^{2}+\left(\sum_{1 \leqslant i \neq j \leqslant k-1}\left|\left\langle x_{i}, x_{j}\right\rangle\right|^{2}\right)^{1 / 2}}\right) \\
& \leqslant \prod_{j=1}^{n}\left\|x_{j}\right\|^{2} .
\end{aligned}
$$

Further on, if we choose $\alpha_{i}=\left\langle x, x_{i}\right\rangle, z_{i}=x_{i}, i \in\{1, \ldots, n\}$ in (2.11), then we may state the inequality

$$
\left\|\sum_{i=1}^{n}\left\langle x, x_{i}\right\rangle x_{i}\right\|^{2} \leqslant \sum_{i=1}^{n}\left|\left\langle x, x_{i}\right\rangle\right|^{2}\left(\max _{1 \leqslant i \leqslant n}\left\|x_{i}\right\|^{2}+(n-1) \max _{1 \leqslant i \neq j \leqslant n}\left|\left\langle x_{i}, x_{j}\right\rangle\right|\right) .
$$

Utilising (2.19) and (2.16) we may also state the following result.

Theorem 3. Let $\left\{x_{1}, \ldots, x_{n}\right\}, M$ and $x$ be as in Theorem 1. Then

$$
d^{2}(x, M) \leqslant \frac{\|x\|^{2}\left[\max _{1 \leqslant i \leqslant n}\left\|x_{i}\right\|^{2}+(n-1) \max _{1 \leqslant i \neq j \leqslant n} \mid\left\langle x_{i}, x_{j}\right\rangle \|\right]-\sum_{i=1}^{n}\left|\left\langle x, x_{i}\right\rangle\right|^{2}}{\max _{1 \leqslant i \leqslant n}\left\|x_{i}\right\|^{2}+(n-1) \max _{1 \leqslant i \neq j \leqslant n}\left|\left\langle x_{i}, x_{j}\right\rangle\right|}
$$

or, equivalently,

$$
\begin{aligned}
& \Gamma\left(x_{1}, \ldots, x_{n}, x\right)\|x\|^{2}\left[\max _{1 \leqslant i \leqslant n}\left\|x_{i}\right\|^{2}+(n-1) \max _{1 \leq i \neq j \leqslant n} \mid\left\langle x_{i}, x_{j}\right\rangle \|\right]-\sum_{i=1}^{n}\left|\left\langle x, x_{i}\right\rangle\right|^{2} \\
& \max _{1 \leqslant i \leqslant n}\left\|x_{i}\right\|^{2}+(n-1) \max _{1 \leqslant i \neq j \leqslant n}\left|\left\langle x_{i}, x_{j}\right\rangle\right| \\
& \times \Gamma\left(x_{1}, \ldots, x_{n}\right) .
\end{aligned}
$$


REMARK 4. The above result (2.20) provides a refinement for the following generalisation of Bessel's inequality:

$$
\sum_{i=1}^{n}\left|\left\langle x, x_{i}\right\rangle\right|^{2} \leqslant\|x\|^{2}\left[\max _{1 \leqslant i \leqslant n}\left\|x_{i}\right\|^{2}+(n-1) \max _{1 \leqslant i \neq j \leqslant n}\left|\left\langle x_{i}, x_{j}\right\rangle\right|\right],
$$

obtained by the author in [7].

One can also provide the corresponding refinement of Hadamard's inequality (1.4) on using (2.21), that is,

$$
\begin{aligned}
& \Gamma\left(x_{1}, \ldots, x_{n}\right) \\
& \quad \leqslant\left\|x_{1}\right\|^{2} \prod_{k=2}^{n}\left(\left\|x_{k}\right\|^{2}-\frac{\sum_{i=1}^{k-1}\left|\left\langle x_{k}, x_{i}\right\rangle\right|^{2}}{\max _{1 \leqslant i \leqslant k-1}\left\|x_{i}\right\|^{2}+(k-2) \max _{1 \leqslant i \neq j \leqslant k-1}\left|\left\langle x_{i}, x_{j}\right\rangle\right|}\right) \\
& \leqslant \prod_{j=1}^{n}\left\|x_{j}\right\|^{2} .
\end{aligned}
$$

3. OTHER UPPER BOUNDS FOR $d(x, M)$

In $[8$, p. 140$]$ the author obtained the following inequality that is similar to the Cauchy-Bunyakovsky-Schwarz result.

LEmmA 2. Let $z_{1}, \ldots, z_{n} \in H$ and $\alpha_{1}, \ldots, \alpha_{n} \in \mathbb{K}$. Then one has the inequalities:

$$
\begin{aligned}
\left\|\sum_{i=1}^{n} \alpha_{i} z_{i}\right\|^{2} & \leqslant \sum_{i=1}^{n}\left|\alpha_{i}\right|^{2} \sum_{j=1}^{n}\left|\left\langle z_{i}, z_{j}\right\rangle\right| \\
& \leqslant\left\{\begin{array}{l}
\sum_{i=1}^{n}\left|\alpha_{i}\right|^{2} \max _{1 \leqslant i \leqslant n}\left[\sum_{j=1}^{n}\left|\left\langle z_{i}, z_{j}\right\rangle\right|\right] \\
\left(\sum_{i=1}^{n}\left|\alpha_{i}\right|^{2 p}\right)^{1 / p}\left(\sum_{i=1}^{n}\left(\sum_{j=1}^{n}\left|\left\langle z_{i}, z_{j}\right\rangle\right|\right)^{q}\right)^{1 / q} \\
\text { where } p>1, \frac{1}{p}+\frac{1}{q}=1 \\
\max _{1 \leqslant i \leqslant n}\left|\alpha_{i}\right|^{2} \sum_{i, j=1}^{n}\left|\left\langle z_{i}, z_{j}\right\rangle\right| .
\end{array}\right.
\end{aligned}
$$

We can state and prove now another upper bound for the distance $d(x, M)$ as follows.

Theorem 4. Let $\left\{x_{1}, \ldots, x_{n}\right\}, M$ and $x$ be as in Theorem 1. Then

$$
d^{2}(x, M) \leqslant \frac{\|x\|^{2} \max _{1 \leqslant i \leqslant n}\left[\sum_{j=1}^{n}\left|\left\langle x_{i}, x_{j}\right\rangle\right|\right]-\sum_{i=1}^{n}\left|\left\langle x, x_{i}\right\rangle\right|^{2}}{\max _{1 \leqslant i \leqslant n}\left[\sum_{j=1}^{n} \mid\left\langle x_{i}, x_{j}\right\rangle \|\right]}
$$

or, equivalently,

$$
\Gamma\left(x_{1}, \ldots, x_{n}, x\right) \leqslant \frac{\|x\|^{2} \max _{1 \leqslant i \leqslant n}\left[\sum_{j=1}^{n} \mid\left\langle x_{i}, x_{j}\right\rangle \|\right]-\sum_{i=1}^{n}\left|\left\langle x, x_{i}\right\rangle\right|^{2}}{\max _{1 \leqslant i \leqslant n}\left[\sum_{j=1}^{n} \mid\left\langle x_{i}, x_{j}\right\rangle \|\right]} \cdot \Gamma\left(x_{1}, \ldots, x_{n}\right) .
$$


ProOF: Utilising the first branch in (3.1) we may state that

$$
\left\|\sum_{i=1}^{n}\left\langle x, x_{i}\right\rangle x_{i}\right\|^{2} \leqslant \sum_{i=1}^{n}\left|\left\langle x, x_{i}\right\rangle\right|^{2} \max _{1 \leqslant i \leqslant n}\left[\sum_{j=1}^{n}\left|\left\langle x_{i}, x_{j}\right\rangle\right|\right]
$$

for any $x \in H$.

Now, since, by the representation formula (1.3) we have

$$
d^{2}(x, M)=\|x\|^{2}-\frac{\sum_{i=1}^{n}\left|\left\langle x, x_{i}\right\rangle\right|^{2}}{\left\|\sum_{i=1}^{n}\left\langle x, x_{i}\right\rangle x_{i}\right\|^{2}} \cdot \sum_{i=1}^{n}\left|\left\langle x, x_{i}\right\rangle\right|^{2},
$$

for $x \notin M^{\perp}$, hence, by (3.4) and (3.5) we deduce the desired result (3.2).

REMARK 5. In 1971, Bombieri [3] proved the following generalisation of Bessel's inequality, however not stated in the general form for inner products. The general version can be found for instance in [9, p. 394]. It reads as follows: if $y, y_{1}, \ldots, y_{n}$ are vectors in the inner product space $(H ;\langle\cdot, \cdot\rangle)$, then

$$
\sum_{i=1}^{n}\left|\left\langle y, y_{i}\right\rangle\right|^{2} \leqslant\|y\|^{2} \max _{1 \leqslant i \leqslant n}\left\{\sum_{j=1}^{n}\left|\left\langle y_{i}, y_{j}\right\rangle\right|\right\} .
$$

Obviously, when $\left\{y_{1}, \ldots, y_{n}\right\}$ are orthonormal, the inequality (3.6) produces Bessel's inequality.

In this respect, we may regard our result (3.2) as a refinement of the Bombieri inequality (3.6).

REMARK 6. On making use of a similar argument to that in Remark 1, we obtain the following refinement for the Hadamard inequality:

$$
\begin{aligned}
\Gamma\left(x_{1}, \ldots, x_{n}\right) & \leqslant\left\|x_{1}\right\|^{2} \prod_{k=2}^{n}\left[\left\|x_{k}\right\|^{2}-\frac{\sum_{i=1}^{k-1}\left|\left\langle x_{k}, x_{i}\right\rangle\right|^{2}}{\left.\max _{1 \leqslant i \leqslant k-1}\left|\sum_{j=1}^{k-1}\right|\left\langle x_{i}, x_{j}\right\rangle \mid\right]}\right] \\
& \leqslant \prod_{j=1}^{n}\left\|x_{j}\right\|^{2} .
\end{aligned}
$$

Another different Cauchy-Bunyakovsky-Schwarz type inequality is incorporated in the following lemma [6].

LEMMA 3. Let $z_{1}, \ldots, z_{n} \in H$ and $\alpha_{1}, \ldots, \alpha_{n} \in \mathbb{K}$. Then

$$
\left\|\sum_{i=1}^{n} \alpha_{i} z_{i}\right\|^{2} \leqslant\left(\sum_{i=1}^{n}\left|\alpha_{i}\right|^{p}\right)^{2 / p}\left(\sum_{i, j=1}^{n}\left|\left\langle z_{i}, z_{j}\right\rangle\right|^{q}\right)^{1 / q}
$$

for $p>1,1 / p+1 / q=1$.

If in (3.8) we choose $p=q=2$, then we get

$$
\left\|\sum_{i=1}^{n} \alpha_{i} z_{i}\right\|^{2} \leqslant \sum_{i=1}^{n}\left|\alpha_{i}\right|^{2}\left(\sum_{i, j=1}^{n}\left|\left\langle z_{i}, z_{j}\right\rangle\right|^{2}\right)^{1 / 2} .
$$


Based on (3.9), we can state the following result that provides yet another upper bound for the distance $d(x, M)$.

THEOREM 5. Let $\left\{x_{1}, \ldots, x_{n}\right\}, M$ and $x$ be as in Theorem 1. Then

$$
d^{2}(x, M) \leqslant \frac{\|x\|^{2}\left(\sum_{i, j=1}^{n}\left|\left\langle x_{i}, x_{j}\right\rangle\right|^{2}\right)^{1 / 2}-\sum_{i=1}^{n}\left|\left\langle x, x_{i}\right\rangle\right|^{2}}{\left(\sum_{i, j=1}^{n}\left|\left\langle x_{i}, x_{j}\right\rangle\right|^{2}\right)^{1 / 2}}
$$

or, equivalently,

$$
\Gamma\left(x_{1}, \ldots, x_{n}, x\right) \leqslant \frac{\|x\|^{2}\left(\sum_{i, j=1}^{n}\left|\left\langle x_{i}, x_{j}\right\rangle\right|^{2}\right)^{1 / 2}-\sum_{i=1}^{n}\left|\left\langle x, x_{i}\right\rangle\right|^{2}}{\left(\sum_{i, j=1}^{n}\left|\left\langle x_{i}, x_{j}\right\rangle\right|^{2}\right)^{1 / 2}} \cdot \Gamma\left(x_{1}, \ldots, x_{n}\right) .
$$

Similar comments apply related to Hadamard's inequality. We omit the details.

\section{Some Conditional Bounds}

In the recent paper [5], the author has established the following reverse of the Bessel inequality.

Let $(H ;\langle\cdot, \cdot\rangle)$ be an inner product space over the real or complex number field $\mathbb{K}$, $\left\{e_{i}\right\}_{i \in I}$ a finite family of orthonormal vectors in $H, \varphi_{i}, \phi_{i} \in \mathbb{K}, i \in I$ and $x \in H$. If

$$
\operatorname{Re}\left\langle\sum_{i \in I} \phi_{i} e_{i}-x, x-\sum_{i \in I} \varphi_{i} e_{i}\right\rangle \geqslant 0
$$

or, equivalently,

$$
\left\|x-\sum_{i \in I} \frac{\varphi_{i}+\phi_{i}}{2} e_{i}\right\| \leqslant \frac{1}{2}\left(\sum_{i \in I}\left|\phi_{i}-\varphi_{i}\right|^{2}\right)^{1 / 2}
$$

then

$$
(0 \leqslant)\|x\|^{2}-\sum_{i \in I}\left|\left\langle x, e_{i}\right\rangle\right|^{2} \leqslant \frac{1}{4} \sum_{i \in I}\left|\phi_{i}-\varphi_{i}\right|^{2} .
$$

The constant $1 / 4$ is best possible in the sense that it cannot be replaced by a smaller quantity.

THEOREM 6. Let $\left\{x_{1}, \ldots x_{n}\right\}$ be a linearly independent system of vectors in $H$ and $M:=\operatorname{span}\left\{x_{1}, \ldots x_{n}\right\}$. If $\gamma_{i}, \Gamma_{i} \in \mathbb{K}, i \in\{1, \ldots, n\}$ and $x \in H \backslash M^{\perp}$ is such that

$$
\operatorname{Re}\left\langle\sum_{i=1}^{n} \Gamma_{i} x_{i}-x, x-\sum_{i=1}^{n} \gamma_{i} x_{i}\right\rangle \geqslant 0,
$$

then we have the bound

$$
d^{2}(x, M) \leqslant \frac{1}{4}\left\|\sum_{i=1}^{n}\left(\Gamma_{i}-\gamma_{i}\right) x_{i}\right\|^{2}
$$


or, equivalently,

$$
\Gamma\left(x_{1}, \ldots, x_{n}, x\right) \leqslant \frac{1}{4}\left\|\sum_{i=1}^{n}\left(\Gamma_{i}-\gamma_{i}\right) x_{i}\right\|^{2} \Gamma\left(x_{1}, \ldots, x_{n}\right) .
$$

Proof: It is easy to see that in an inner product space for any $x, z, Z \in H$ one has

$$
\left\|x-\frac{z+Z}{2}\right\|^{2}-\frac{1}{4}\|Z-z\|^{2}=\operatorname{Re}\langle Z-x, x-z\rangle,
$$

therefore, the condition (4.4) is actually equivalent to

$$
\left\|x-\sum_{i=1}^{n} \frac{\Gamma_{i}+\gamma_{i}}{2} x_{i}\right\|^{2} \leqslant \frac{1}{4}\left\|\sum_{i=1}^{n}\left(\Gamma_{i}-\gamma_{i}\right) x_{i}\right\|^{2} .
$$

Now, obviously,

$$
d^{2}(x, M)=\inf _{y \in M}\|x-y\|^{2} \leqslant\left\|x-\sum_{i=1}^{n} \frac{\Gamma_{i}+\gamma_{i}}{2} x_{i}\right\|^{2}
$$

and thus, by (4.7) and (4.8) we deduce (4.5).

The last inequality is obvious by the representation (1.2).

REMARK 7. Utilising various Cauchy-Bunyakovsky-Schwarz type inequalities we may obtain more convenient (although coarser) bounds for $d^{2}(x, M)$. For instance, if we use the inequality (2.11) we can state the inequality:

$$
\left\|\sum_{i=1}^{n}\left(\Gamma_{i}-\gamma_{i}\right) x_{i}\right\|^{2} \leqslant \sum_{i=1}^{n}\left|\Gamma_{i}-\gamma_{i}\right|^{2}\left(\max _{1 \leqslant i \leqslant n}\left\|x_{i}\right\|^{2}+(n-1) \max _{1 \leqslant i<j \leqslant n}\left|\left\langle x_{i}, x_{j}\right\rangle\right|\right),
$$

giving the bound:

$$
d^{2}(x, M) \leqslant \frac{1}{4} \sum_{i=1}^{n}\left|\Gamma_{i}-\gamma_{i}\right|^{2}\left[\max _{1 \leqslant i \leqslant n}\left\|x_{i}\right\|^{2}+(n-1) \max _{1 \leqslant i<j \leqslant n}\left|\left\langle x_{i}, x_{j}\right\rangle\right|\right],
$$

provided (4.4) holds true.

Obviously, if $\left\{x_{1}, \ldots, x_{n}\right\}$ is an orthonormal family in $H$, then from (4.9) we deduce the reverse of Bessel's inequality incorporated in (4.3).

If we use the inequality (2.12), then we can state the inequality

$$
\left\|\sum_{i=1}^{n}\left(\Gamma_{i}-\gamma_{i}\right) x_{i}\right\|^{2} \leqslant \sum_{i=1}^{n}\left|\Gamma_{i}-\gamma_{i}\right|^{2}\left[\max _{1 \leqslant i \leqslant n}\left\|x_{i}\right\|^{2}+\left(\sum_{1 \leqslant i \neq j \leqslant n}\left|\left\langle x_{i}, x_{j}\right\rangle\right|^{2}\right)^{1 / 2}\right],
$$

giving the bound

$$
d^{2}(x, M) \leqslant \frac{1}{4} \sum_{i=1}^{n}\left|\Gamma_{i}-\gamma_{i}\right|^{2}\left[\max _{1 \leqslant i \leqslant n}\left\|x_{i}\right\|^{2}+\left(\sum_{1 \leqslant i \neq j \leqslant n}\left|\left\langle x_{i}, x_{j}\right\rangle\right|^{2}\right)^{1 / 2}\right],
$$


provided (4.4) holds true.

In this case, when one assumes that $\left\{x_{1}, \ldots, x_{n}\right\}$ is an orthonormal family of vectors, then (4.10) reduces to (4.3) as well.

Finally, on utilising the first branch of the inequality (3.1), we can state that

$$
d^{2}(x, M) \leqslant \frac{1}{4} \sum_{i=1}^{n}\left|\Gamma_{i}-\gamma_{i}\right|^{2} \max _{1 \leqslant i \leqslant n}\left[\sum_{j=1}^{n}\left|\left\langle x_{i}, x_{j}\right\rangle\right|\right]
$$

provided (4.4) holds true.

This inequality is also a generalisation of (4.3).

\section{REFERENCES}

[1] R. Bellman, 'Almost orthogonal series', Bull. Amer. Math. Soc. 50 (1944), 517-519.

[2] R.P. Boas, 'A general moment problem', Amer. J. Math. 63 (1941), 361-370.

[3] E. Bombieri, 'A note on the large sieve', Acta Arith. 18 (1971), 401-404.

[4] F. Deutsch, Best Approximation in inner product spaces, CMS Books in Mathematics (Springer-Verlag, New York, Berlin, Heidelberg, 2001).

[5] S.S. Dragomir, 'A counterpart of Bessel's inequality in inner product spaces and some Grüss type related results', RGMIA Res. Rep. Coll. 6 (2003). Supplement, Article 10. [Online: http://rgmia.vu.edu.au/v6(E) .htm1].

[6] S.S. Dragomir, 'Some Bombieri type inequalities in inner product spaces', J. Indones. Math. Soc. 10 (2004), 91-97.

[7] S.S. Dragomir, 'On the Boas-Bellman inequality in inner product spaces', Bull. Austral. Math. Soc. 69 (2004), 217-225.

[8] S.S. Dragomir, 'Advances in inequalities of the Schwarz, Grüss and Bessel type in inner product spaces', RGMIA Monographs, Victoria University (2004). [Online: http://rgmia.vu.edu.au/monographs/advances.htm].

[9] D.S. Mitrinović, J.E. Pečarić and A.M. Fink, Classical and new inequalities in analysis (Kluwer Academic, Dordrecht, 1993).

School of Computer Science and Mathematics

Victoria University of Technology

PO Box 14428

MCMC, Vic 8001

Australia

e-mail: sever@csm.vu.edu.au 